\title{
Low Doses of Ionized Radiation and Hypomagnetic Field Alter Redox Properties of Water and Physiological Characteristics of Seeds of the Highest Plants
}

\author{
Svetlana Stepanovna Moisa, Vladimir Vladimirovich Tsetlin, \\ Margarita Alexandrovna Levinskich, Elena Leonidovna Nefedova \\ Federal State-Financed Establishment of Science, State Scientific Center of the Russian Federation, \\ Institute of Biomedical Problems of the Russian Academy of Sciences, Moscow, Russia \\ Email: butalana07@list.ru
}

Received 13 April 2016; accepted 8 July 2016; published 12 July 2016

Copyright @ 2016 by authors and Scientific Research Publishing Inc. This work is licensed under the Creative Commons Attribution International License (CC BY). http://creativecommons.org/licenses/by/4.0/

cC) (i) Open Access

\begin{abstract}
The influence of a 40-fold attenuated geomagnetic field and its combined action with low doses of $\alpha$ - and $\gamma$-irradiation on the physiological characteristics of seeds of the highest plants and redox properties of water was investigated. It established the reduction of seed germination both under direct and indirect effects due to water action of attenuated geomagnetic field. A negative effect of hypomagnetic field on grown characteristics of seeds under indirect effect via water was decreased by the low doses of $\gamma$-irradiation, and was increased by low doses of $\alpha$-irradiation, i.e. ionized radiation was the dominant factor in their combined action. It was revealed the increasing of the value of the oxidation-reduction potential of water under the influence of low-intensive $\alpha$-irradiation $\left({ }^{239} \mathrm{Pu}\right), \gamma$-irradiation $\left({ }^{137} \mathrm{Cs}\right)$ and also that the magnetic induction attenuated pointing to a natural decline. The increasing of the oxidation-reduction potential value testifies about "the regular decreasing of internal energy of water molecules" and the increasing of its oxidative properties, which, in our opinion, is caused the inhibition of the germination of seeds. It is supposed that namely water is the main component in the effects of studying factors on bio-objects, which acts due to the alterations of the properties and structural content of water.
\end{abstract}

\section{Keywords}

$\alpha$-and $\gamma$-Irradiation, Hypomagnetic Field, Oxidation-Reduction Potential of Water, Physiological Characteristics of Seeds of the Highest Plants 


\section{Introduction}

One of the constantly effecting factors on living organisms is geomagnetic field of Earth. And, although geomagnetic field doesn't stay constant both to induction and to configuration [1], its sharp changing isn't the factor of natural selection, animal reaction on it isn't fast genetically and can be the most diverse. Our previous investigations showed the influence of hypomagnetic field (HMF) on the embryonic development of great ramshorn Planorbarius corneus [2]. Another important factor is Ionized Radiation (IR) of the different types. Effect of IR on living organism in the doses of 30 - 50 sGy was studied on the different biological objects [3]-[8]. At the same time the effect of ionized radiation in the doses not more than 10 sGy on living organisms remains studying; little and common regularities of their influence on biological objects are unknown. The question about the ratio of the influences on organism direct effect of IR and influence of changing environment due to this factor is not clear [9]. Earlier model experiments [2] [10] [11] were by established changing oxidation-reduction properties of water under the effects of environment factors ("electromagnetic" background, IR, HMF, under eruptions of volcano, solar eclipse). Proceed from these ideas, it is logically to investigate, how water properties change under the effect of character for cosmic flight low doses of IR and another factor of cosmic spaceweakening geomagnetic field and also their combined action. In this respect a special interest is the studying of such water property as oxidation-reduction potential (ORP) and hydrogen parameter (pH). ORP should consider as a homeostatic characteristic of biological liquids of organism [12]. As biological object we chose the seeds of the highest plants.

The purpose of this investigation is to study the effect of IR in low doses, weakening geomagnetic field and their combined action on water reaction and physiological characteristics of the highest plants seeds.

\section{Methods}

Two groups of experiments were carried out: the effect of weakening of magnetic field and combined action with low doses of IR on physiological characteristics of the highest plants seeds and the effect of IR in low doses, HMF and its combined action on redox properties of water.

\subsection{Effect of Weakening of Magnetic Field and Combined Action with Low Doses of Ionized Radiation on Rising and Germination of Seeds}

In the first group of experiments were studied 4 series. Airy dry certificated seeds of radishes Raphanus sativus L: pink-red (firm "Sedek”), radishes Cherry Bomb (firm "Burpee”), radishes “Sango", Red Pepper sweet Capsicum annuum (firm "Burpee”) and fenugreek Trigonella foenum graecum L. (firm "Thompson and Morgan”) served as the objects of investigation, which for their morpho-physiological features relate to the highest plants because of they are Magnoliophyta, or Angiospermae.

The radishes dry seeds and red pepper seeds (50 ones) were put in three cup of Petri (diameter $4 \mathrm{~cm}$ ) on 2 layers of filter paper, wet with settled during a day water-piped water and stayed to grow in normal geomagnetic field (8 days). It served as control (1-st series). In the 2-nd series, prepared analogically control series, the cups of Petri were put in the chamber with a 40 -fold attenuated geomagnetic field ( $1 \mathrm{mkT})$ during 8 days. In the 3-rd series the radishes dry seeds wet with settled during a day water-piped water, preliminary put for 30 minutes in the chamber with weakening geomagnetic field, as far as water condition, undergoing to outer influence, is formed not moment, but 15 - 30 minutes later, when the net of hydrogen links are formed [13]. Then the seeds were stayed for the germination under normal geomagnetic field. In the 4-th series dry seeds of radishes "Sango" and fenugreek Trigonella foenum graecum wet in water, preliminary undergoing to $\alpha$ - and $\gamma$-radiation (power of absorbed dose conformed to the radiation situation in space apparatus), and set in hypomagnetic chamber (HMC) -the device "Screen ferromagnetic", produced in Zelenograd, Moscow region. A 40-fold attenuated geomagnetic field was reached in HMC, which represented a cylinder, making from 15 lays of permaloj, inner diameter $=110 \mathrm{~mm}, \mathrm{~L}=500 \mathrm{~mm}$. A uniformness of weakening of magnetic field along the chamber length reached with the help of creating wall thickness of chamber to the point of $15 \mathrm{~mm}$ and controlled due to magnetometer.

\subsection{Effect of Ionized Radiation on Water}

Method of Radiation Processing of Water. Radiation processing of water-piped water, preliminary settled during a day was carried out under the effect of $\alpha$ - and $\gamma$-particles of natural radio-nuclides ${ }^{239} \mathrm{Pu}$ and ${ }^{137} \mathrm{Cs}$. The open 
surface of water in separate vessels is undergone to the action of each type of particles. The radiation of water in the absent of biological objects were carried out under room temperature $22^{\circ} \mathrm{C}-24^{\circ} \mathrm{C}$ in chemical glasses of 200 $\mathrm{ml}$ volume. The source of irradiation was stayed on the glass close to water surface (but without direct contact with water surface). The time of exposition of each portion of water was 20 minutes. The absorbed dose of $\alpha$ radiation with the energy 5, $15 \mathrm{MeV}$ isn't enhance for the exposition $8 \mathrm{sGy}$ under the thickness of radiation layer of water $\sim 45 \mathrm{mkm}$. In one's turn, under the influence of $\gamma$-quantum (maximum energy $660 \mathrm{keV}$ ) accumulated dose was 20 - 25 sGy under the dose power-690 $\mu \mathrm{R} / \mathrm{h}$.

The duration of the experiments consisted of 7 days.

\subsection{Effect of Low Doses of Ionized Radiation and Weakening of Magnetic Field on ORP and $\mathrm{pH}$ Value of Water}

In the second group of experiments the influence of IR in low doses and the weakening in a 40-fold attenuated geomagnetic field on the oxidation-reduction properties of water, detecting ORP and pH value of water with the help of ionomer И-160МИ. It was fulfilled 4 series of experiments.

In the 1-st series it was studied the effect of low doses of radiation of $\alpha$-particles (activity-15 kBk, accounts energy density-6.7 $\times 10^{-8} \mathrm{~W} / \mathrm{cm}^{2}$ ) on the ORP value. In the 2-nd series it was studied the effect of low doses of radiation of $\gamma$-quantum on the ORP value. Natural radiation background during the carrying out of experiments consisted of $37 \mu \mathrm{R} / \mathrm{h}$. The radiation of water was fulfilled by the increasing doses (the time of exposition started from 2 to 20 minutes). In the 3-rd series it was investigated the effect of a 40-fold attenuated geomagnetic field $(\sim 1.0 \mathrm{mkT})$ on the ORP value. In the 4-th series it was carried out the investigation of the effect of combined action of IR in low doses ( $\alpha$ - and $\gamma$-radiation) and HMF on ORP value and $\mathrm{pH}$. With this aim three samples of water were taken, which were preliminary measured ORP value and $\mathrm{pH}$, then the second sample of water was undergone to the radiation by $\alpha$-particles, the third- $\gamma$-quantum, the time of exposition was 20 minutes, the first sample served control. After that all samples were set in HMC for 8 days. In 8 days in the investigating samples of water it was measured ORP value and $\mathrm{pH}$.

The results of experiments was undergone the standard methods of statistics. The estimation of statistic significance of the parameters and differences of the compared selections was carried out according to the Student's criterion on the reliability level below $\mathrm{P} \leq 0.05$.

\section{Results and Discussion}

\subsection{Dry Seeds of the Highest Plant}

\subsubsection{Effect of Weakening of Magnetic Field on Rising and Germination of Seeds}

In normal geomagnetic field with the magnetic induction 40 - $43 \mathrm{mkT}$ (control) the number of growing seeds of red pepper consisted of $25 \pm 2$; pink-red radishes-16 \pm 1 ; Cherry Bomb radishes-23 \pm 2 ones. The length of counterfoil consisted of $2.84 \pm 0.5 \mathrm{~cm}, 2.66 \pm 0.3 \mathrm{~cm}$ and $5.10 \pm 1.0 \mathrm{~cm}$, the length of hypocotile-3.54 $\pm 0.4 \mathrm{~cm}$, $1.85 \pm 0.2 \mathrm{~cm}$ and $3.40 \pm 0.6 \mathrm{~cm}$, correspondently, that was in the range of norm for the parameters of physiological characteristics of these kinds of the highest plants seeds (Table 1, Table 2).

Under the weakening geomagnetic field (the 2-nd series) it was noticed the decreasing of germination energy of the radishes and red pepper seeds for $23 \%$ and $37 \%$, correspondently, in comparison with the control series. The development of the sprouts of two kinds of the radishes and red pepper significantly slowed down, that it was found their reflex in the decreasing of the length of counterfoil and hypocotile in 4 - 5 times and in 2.5 - 5.5 times, correspondently, in comparison with control (Table 1, Table 2).

Table 1. The rising of seeds and growing characteristics of red pepper sprouts in the conditions of normal (control) and the weakening geomagnetic field $(\mathrm{x} \pm \mathrm{S})$.

\begin{tabular}{ccc} 
Parameters & Control & HMF, mkT \\
\hline Number of growing seeds & $25 \pm 2$ & $9 \pm 1^{*}$ \\
The length of counterfoil $(\mathrm{cm})$ & $2.84 \pm 0.5$ & $1.14 \pm 0.2^{*}$ \\
The length of hypocotile $(\mathrm{cm})$ & $3.54 \pm 0.4$ & $0.65 \pm 0.1^{*}$ \\
\hline
\end{tabular}

Note: *the reliability of results differences $(\mathrm{P} \leq 0.05)$. 
Table 2. The comparison of physiological characteristics of radishes seeds depending on germination condition $(x \pm S)$.

\begin{tabular}{|c|c|c|c|c|c|c|}
\hline \multirow[b]{2}{*}{ Parameters } & \multicolumn{3}{|c|}{ Pink-red radishes } & \multicolumn{3}{|c|}{ Cherry bomb radishes } \\
\hline & $\begin{array}{l}\text { Geomagnet. } \\
\text { Field (control) }\end{array}$ & $\begin{array}{l}\text { HMF (direct } \\
\text { action) }\end{array}$ & $\begin{array}{c}\text { Geomagnet. } \\
\text { Field (indirect } \\
\text { effect of HMF via } \\
\text { water) }\end{array}$ & $\begin{array}{l}\text { Geomagnit. Field } \\
\text { (control) }\end{array}$ & $\begin{array}{l}\text { HMF (direct } \\
\text { action) }\end{array}$ & $\begin{array}{l}\text { Geomagnet. } \\
\text { Field (indirect } \\
\text { effect of HMF } \\
\text { via water) }\end{array}$ \\
\hline $\begin{array}{c}\text { The rising } \\
\text { of seeds (ones) }\end{array}$ & $16 \pm 1$ & $13 \pm 1$ & $14 \pm 1$ & $23 \pm 2$ & $17 \pm 1^{*}$ & $18 \pm 1^{*}$ \\
\hline $\begin{array}{l}\text { The length of } \\
\text { counterfoil }(\mathrm{cm})\end{array}$ & $2.66 \pm 0.4$ & $0.60 \pm 0.1^{*}$ & $0.73 \pm 0.1^{*}$ & $5.10 \pm 1.0$ & $1.06 \pm 0.2^{*}$ & $1.60 \pm 0.2^{*}$ \\
\hline $\begin{array}{l}\text { The length of } \\
\text { hypocotile }(\mathrm{cm})\end{array}$ & $1.85 \pm 0.2$ & $0.45 \pm 0.05 *$ & $0.60 \pm 0.1^{*}$ & $3.40 \pm 0.6$ & $0.65 \pm 0.1^{*}$ & $1.20 \pm 0.1^{*}$ \\
\hline
\end{tabular}

Note: *the reliability of results differences $(\mathrm{P} \leq 0.05)$.

Indirect due to the water, which was preliminary undergone to exposure during 30 minutes in the conditions of a 40-fold attenuated geomagnetic field, the effect of the weakening geomagnetic field was also affected on the rising of seeds and the development of the sprouts of two kinds of radishes (3-rd series). So, the numbers of the rising seeds of two kinds of radishes was less $15 \%-25 \%$ of the value in control investigations and reliable didn't differ from the same value in the 2-nd series. The values of the length of counterfoil and hypocotile of the seeds in the 3-rd series were some more than the parameters in the seeds of the 2-nd series, but also were in 3 times less in comparison with control measurements (Table 2).

Thus, a 40-fold attenuated geomagnetic field both direct and indirect effect due to the water, led to the decreasing of the rising and the development of the sprouts of the seeds of two kinds of radishes.

\subsubsection{Effect of Combined Action of Ionized Radiation and Hypomagnetic Field on} Physiological Characteristics of the Highest Plant Seeds

The influence of HMF in combination with low doses of $\alpha$ - and $\gamma$-irradiation on the seeds of radishes "Sango" and fenugreek led to the changing of the rising of seeds (4-th series). So, in conditions of HMF the rising of the seeds of both cultures on the water, undergoing by $\gamma$-particles, increased $40 \%$, and undergoing by $\alpha$-particles, decreasing $40 \%$ in comparison with the control investigations in normal geomagnetic field. Thus, the rising of the seeds of both cultures, wetting with water, undergoing by $\gamma$-quantum, in the conditions of the weakening of geomagnetic field was more than the parameters in control measurements (Figure 1, Table 3).

In the conditions of the weakening geomagnetic field the length of radishes and fenugreek sprouts, germinating on the water, undergoing by $\gamma$-particles, increased $28 \%-40 \%$, and on the water, undergoing by $\alpha$-particles, decreased $30 \%$ for both cultures in comparison with the data, receiving in the conditions of normal geomagnetic field (control).

Thus, under indirect effect due to the water the low doses of $\gamma$-irradiation lowered, $\alpha$-irradiation enhanced the negative effect of a 40-fold attenuated geomagnetic field on the growing characteristics of the highest plants seeds.

These results allow conclude that indirect effect of the weakening geomagnetic field by virtue of water on the rising and physiological characteristic of the highest plants seeds testifies about that this factor reveals its effect due to the alteration of the properties and condition of water. Besides, the modulation of the effect of the weakening geomagnetic field on the rising and physiological characteristics of the highest plants seeds under the effect of changing properties and condition of the water, induced by the preliminary radiation of $\alpha$ - and $\gamma$-particles, testifies also about that namely the water is that main factor in the receiving effect of the weakening geomagnetic field (and, apparently, as any other factor). The modulation of effect of the weakening geomagnetic field on the rising and physiological characteristics of the highest plant seeds, due to the water, preliminary undergoing to the radiation of $\alpha$ - and $\gamma$-irradiation, testifies and about that the ionized radiation is the dominant factor in the combined effect with the weakening geomagnetic field.

\subsection{Water}

\subsubsection{Effect of Ionized Radiation in Low Doses on Water ORP Value $\alpha$-irradiation}




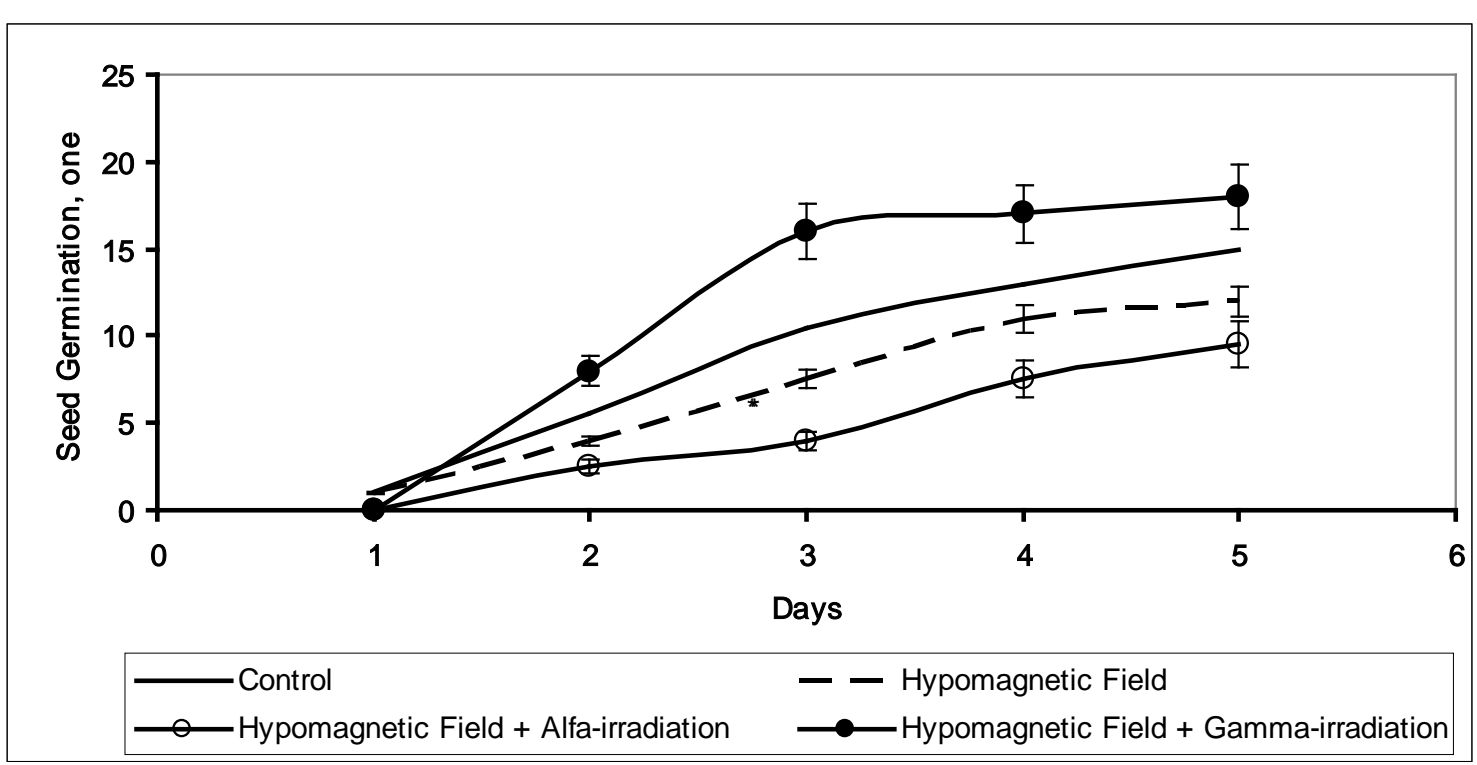

Figure 1. Dynamics of the rate of seed germination radishes "Sango" on the water undergoing $\alpha$ - and $\gamma$-irradiation in hypomagnetic chamber.

Table 3. The length of the risings of Radishes "Sango" and Fenugreek under the seed sprouting in hypomagnetic field on the water, undergoing by $\alpha$ - and $\gamma$-irradiation $(\mathrm{cm}, \mathrm{x} \pm \mathrm{S})$.

\begin{tabular}{cccc}
\hline Agricultural culture & $\alpha$-irradiation + HMF & $\gamma$-irradiation + HMF & Geomagnetic Field (control) \\
\hline & \multicolumn{1}{c}{ The Length of the risings, cm } & $2.5 \pm 0.2$ \\
Radishes "Sango" & $1.80 \pm 0.2^{*}$ & $3.2 \pm 0.4^{*}$ & $3.0 \pm 0.3$ \\
Fenugreek & $2.1 \pm 0.1^{*}$ & $4.2 \pm 0.5^{*}$ & \\
\hline
\end{tabular}

Note: *-the reliability of results differences $(\mathrm{P} \leq 0.05)$.

The value of water ORP, measuring before the start of radiation by $\alpha$-particles, consisted of $265 \mathrm{mV}$. Change of water ORP parameters under the effect of $\alpha$-radiation in range of enhancing doses from 2 to $22 \mathrm{nGy}$ revealed the following type: water ORP, measuring in 10 second after the end of water radiation in the dose of $2 \mathrm{nGy}$, quickly increased to $360 \mathrm{mV}$. Then according to the enhancing of the dose radiation it was observed the same fast decline of ORP value to $320 \mathrm{mV}$ (from 4.4 to $8.9 \mathrm{nGy}$ ) with the following fast increasing of ORP value to $380 \mathrm{mV}$ (Figure 2). Further under the following enhancing of radiation dose in the range from 16 to $22 \mathrm{nGy}$ a gradual fall of ORP value almost normal value $-290 \mathrm{mV}$ noticed. The results of measurements demonstrate a non-linear polymodal (almost sinusoidal) character of ORP value change under the effect of enhancing doses of $\alpha$-irradiation, it is conformed with generally known notion [14].

For the elucidation, that how long it will last out the properties of radiated water, i.e. change of water ORP value under the effect of IR, we thought expedient to measure ORP value later 20 minutes after exposition of each dose.

It was discovered, 20 minutes later after the end of radiation a non-linear almost sinusoidal character of dependence on water ORP and dose radiation, kept as before. The curves were almost equi-distant, i.e. the distance between them under the same meaning of dose oscillated in the range of $45-90 \mathrm{mV}$. Value meaning didn't fall, but even increased average $17 \%$.

\section{$\gamma$-irradiation}

In the time of effecting on the water by $\gamma$-irradiation from radio-nuclide source ${ }^{137} \mathrm{Cs}$ it was revealed other dependence on ORP value and dose radiation (Figure 3). Although non-monotonous this dependence manifested, however, the curve, which was registered 20 minutes later after the radiation, a little differed from the initial curve, which was registered immediately after the radiation. In our opinion, the last fact testifies about that radiation by $\gamma$-quantum, which having more narrow energetic spectrum, provokes lesser and more stable change in water condition. 


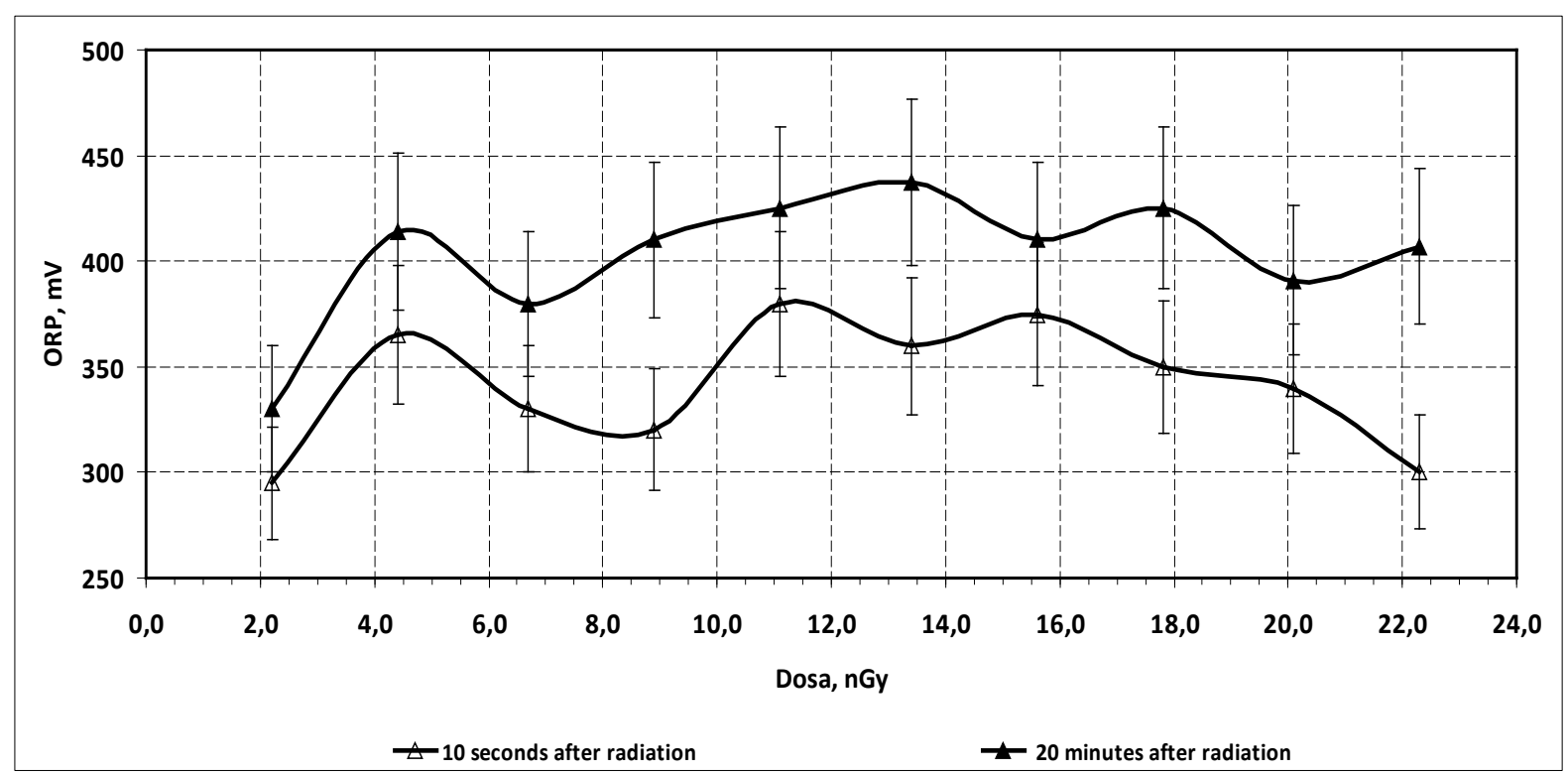

Figure 2. Change of water ORP value under $\alpha$-irradiation.

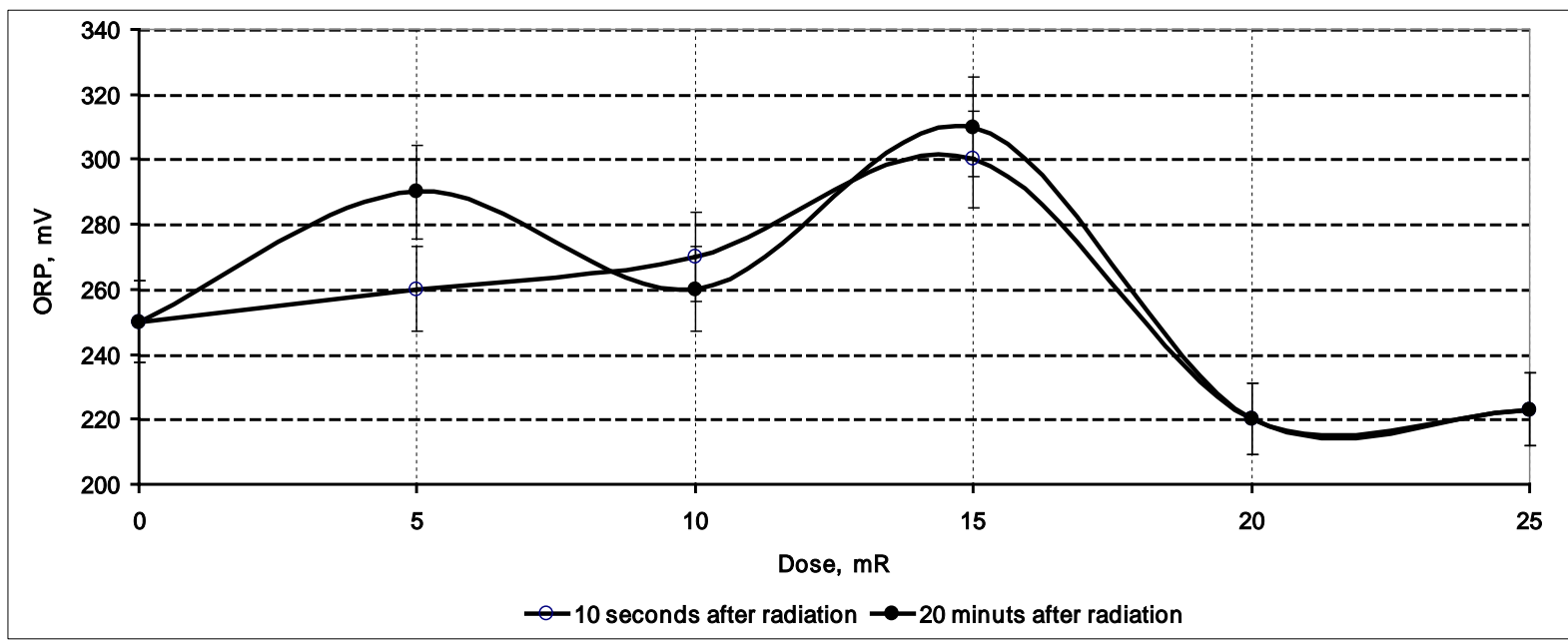

Figure 3. Change of water ORP value under $\gamma$-irradiation.

\subsubsection{Effect of Weakening of Magnetic Field on Water ORP Value}

As the weakening of the induction of magnetic field in HMC the increasing of water ORP value of water medium is revealed (Figure 4), that testifies about naturally determined "decreasing of inner energy of water molecules" [13] and increasing of its oxidative properties. Apparently, in the conditions of the weakening geomagnetic field the energy of electron excitement in water molecules decreases due to the absence of energy pumping from geomagnetic field.

\subsubsection{Change of Water ORP and $\mathrm{pH}$ Value after $\alpha$ - and $\gamma$-Radiation in Hypomagnetic Chamber}

In geomagnetic conditions water ORP consisted of $169 \mathrm{mV}, \mathrm{pH}=7.1$. Eight days later staying in HMC water ORP value increased for $44 \%$ to $243 \mathrm{mV}$, $\mathrm{pH}$ decreased to 5.9 (for $72 \%$ ) for this, i.e. in control water in the conditions of HMF oxidative properties enhanced (Table 4). Thus, hypomagnetic conditions alter redox properties of water, increasing its oxidative ones.

After 20-minute $\alpha$-radiation the parameters of ORP и $\mathrm{pH}$ changed (Table 4). So, ORP value increased for $34 \%$ from 169 to $226 \mathrm{mV}$, pH decreased for 54\% (from 7.1 to 4.6). Hypomagnetic conditions (during 8 days) 


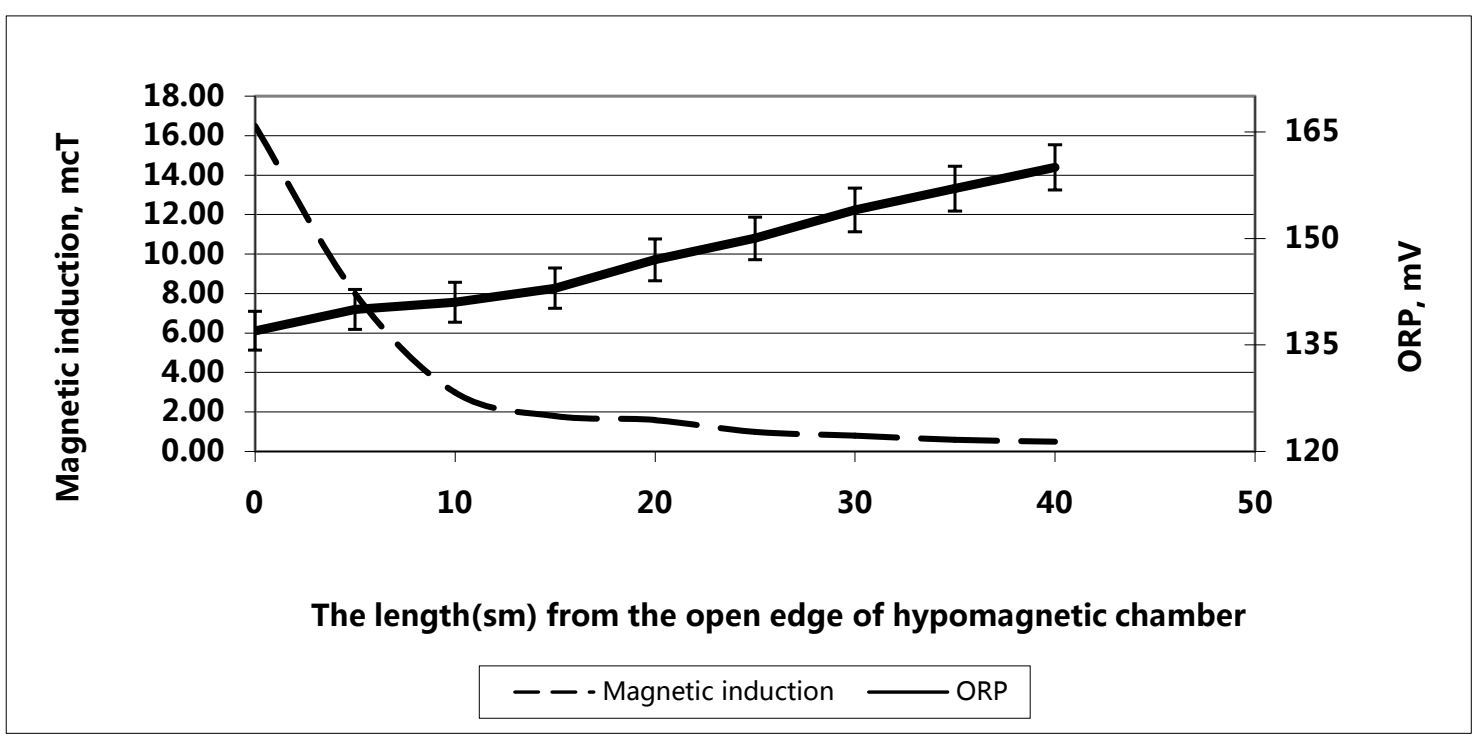

Figure 4. Dependence on ORP value and magnetic flux density.

Table 4. The alterations of water ORP and $\mathrm{pH}$ value after $\alpha$ - and $\gamma$-radiation in hypomagnetic chamber.

\begin{tabular}{ccccccc}
\hline Parameters & $\begin{array}{c}\text { Geomagnet. } \\
\text { Field }\end{array}$ & $\alpha$-radiation & $\gamma$-radiation & HMF+ $\alpha$-radiation & HMF+ $\gamma$-radiation & $\begin{array}{c}\text { HMF water } \\
\text { (control) }\end{array}$ \\
\hline ORP, mV & 169 & 226 & 268 & 260 & 205 & 243 \\
pH & 7.1 & 4.6 & 3.6 & 5.4 & 6.1 & 5.9 \\
\hline
\end{tabular}

enhanced ORP value yet for 15\% (from 226 to $260 \mathrm{mV}$ ), and pH increased for 17\% (from 4.6 to 5.4). Thus, water, undergoing to the radiation of $\alpha$-particles in hypomagnetic conditions still more (for 7\%) enhanced its oxidative properties in comparison with control water in HMC (243 mV in comparison with $260 \mathrm{mV}$ ).

After the radiation of $\gamma$-quantum water ORP value consisted of $268 \mathrm{mV}$, what was for 59\% higher than water ORP in geomagnetic field, $\mathrm{pH}$ value for this fell sharp for 50\% (Table 4). On the 8-th day of water staying in HMC water ORP value decreased for $77 \%$ from 268 to $205 \mathrm{mV}$, and consisted of 84\% from $243 \mathrm{mV}$-ORP value of control water, which was staying during 8 days in $\mathrm{HMC}$, and $\mathrm{pH}$ value, on the contrary, increased for $59 \%$ from 3.6 to 6.1, i.e. in conditions of HMF water, which was undergone to $\gamma$-quantum, gradually restored its properties: oxidative properties decreased, and reduction ones increased (for 21\%). Thus, hypomagnetic conditions affected the water, which was undergone to $\gamma$-radiation, enhancing its reduction properties.

These data allow conclude that the combined action of HMF and radiation in low doses change water oxidation-reduction properties depending on the type and absorb dose of radiation source. In our investigations was shown that in conditions of HMF $\alpha$-irradiation enhanced oxidative, and $\gamma$-quantum-reduction properties of water.

In usual conditions water ORP, measured relatively chlorine-silver electrode comparison, is, as a rule, positive and consists $120 \div 170 \mathrm{mV}$ under the neutral hydrogen parameter $\mathrm{pH}=7.0$.

Under the accommodating for a long time (8 days) glassy bucks with water in HMC, which decreasing the torrent of outer factors-electromagnetic irradiation, and weakening the induction of geomagnetic field in 40 times, the influx of outer energy from geomagnetic field brings down. Apparently, it induces the decreasing of disturbance hydrogen links, the diminution of inner energy of water molecules and the increasing of water electron-acceptor properties, i.e. oxidative capacity of water enhances (increasing of ORP value). Further as a result of electron-exchange interactions between water molecules their dissociation and the increasing of hydrogen ions concentration (the decreasing of $\mathrm{pH}$ value) occurs.

In case of the preliminary activation of water due to ionized radiation, energy influx and excitement of water molecules occurs, i.e. the portion of chemical active molecules with electron-donor properties enhances. In the result of activation the speed of electron-exchange reactions, inducing low-threshold dissociation of the reaction products and the decreasing of $\mathrm{pH}$, enhances [13]. Besides, oxidative-reduction reactions activate under the ef- 
fect of IR on the water and as a result the concentration of active oxygen species increases (rising of ORP value).

In our investigations under the effect of corpuscular $\alpha$-irradiation on the water in the result of break of hydrogen links in molecule associates a great number of initial reagents of oxidative-reduction reactions realize (the increasing of entropy) with a wide range of passing energy to water molecules.

Under the effect of $\gamma$-quantum from mono-energetic source entropy increasing occurs with less range due to more narrow range of energy transfer. Apparently, this peculiarity of energy transfer causes the difference of the properties of investigating water.

The data, receiving in our experiments, testifies about that: first, change of oxidation-reduction properties of water under the effect of enhanced doses of low-intensive $\alpha$-irradiation occurred non-monotonous and non-linear; second, the range of doses, where the decreasing of effect with the increasing of doses, observed for $\alpha$-irradiation is in the range from 4.4 to $8.9 \mathrm{nGy}$; third, change of oxidation-reduction properties of water under the effect of enhanced doses of low-intensive $\alpha$-irradiation occurred polymodal; fourth, oxidation-reduction properties of water, induced by the radiation of enhancing doses of low-intensive $\alpha$-irradiation, were kept 20 minutes later after the time of exposition; fifth, even 20 minutes after the end of radiation exposition by $\alpha$-irradiation a non-linear character was kept; sixth, water ORP values increased average $17 \% 20$ minutes later after the end of the radiation of $\alpha$-irradiation; seventh, change of oxidation-reduction properties of water under the enhanced doses of low-intensive $\gamma$-irradiation occurred with less range of change of ORP values in comparison with the radiation by $\alpha$-irradiation, for mono-energetic $\gamma$-quantum the recession of ORP came, if radiation dose exceeded $1.5 \mathrm{sGy}$; eight, the increasing of water ORP value revealed as magnetic induction attenuated pointing to a natural decline; ninth, in the conditions of HMF water ORP value enhanced, and $\mathrm{pH}$ fell, i.e. oxidative properties of water increased; tenth, under the combined action of HMF and $\alpha$-irradiation oxidative properties of water enhanced; and under the combined action of HMF and $\gamma$-irradiation reduction properties of water increased, that was reflected on the growth characteristics of seeds of the highest plants.

As for the causes of such difference between the results of water radiation by the charged particles and $\gamma$-quantum, one can state the following assumptions, which are based on the thermodynamic [15] regularities of the forming of the distribution of free energy water molecules in their aggregate after the activation by low-intensive ionized irradiation. For the water reaction on the activated effect is characteristic the change of inner energy in the first moment of effect: the increasing of energy of rotating and oscillating levels of water molecules and excitement of energy of electron levels [16]. Further reconstruction of space links of water molecules, forming and destruction of hydrogen links, the forming some dimmers, three-mers and more large associates from water molecules occurs. According to the position of disbalance thermodynamics the accumulated inner energy flow over in the energy of the bound condition of the part of all aggregate of molecules and enthropy decreasing, that can led to the change of free energy of Gibbs [17]. The speed of chemical reactions with the participant of water molecules, going to the practice of theory Gibbs-Arrenius use, determines by free energy of all aggregate of molecules. For the water is characteristic non-stable of links, for example, the origin of "twinkling" clusters. In accordance with this position, in our opinion, one should consider the inhibiting effect of $\gamma$-irradiation and potential effect of $\alpha$-irradiation in their combined action with HMF.

The universal receptor of the fields and amplifier of its action is water, more exactly, water basis of living organisms. Under the effect of a 40-fold attenuated geomagnetic field the electron generation of water molecules decreases (work of electron leaving increases). So long as living organisms are water structures, the amount of water excited molecules, are capable to give back the electrons, decreases and ORP value grows and dissolving water properties change under HMF. ORP characterizes the state of inner biological environment of organism. Apparently, the slowdown of biochemical processes under the sharp weakening of magnetic field from normal level caused the slowdown of seeds growth. In accordance with the position of change water medium state under the effect of HMF and its combined action with IR one should consider, in our opinion, the lowering of rising and slow down of the development of radishes and fenugreek seeds sprouts, what testifies about that, the determine factor of the investigating effects is the change of the water medium state.

\section{Conclusions}

1. A 40-fold attenuated geomagnetic field both under direct and indirect effect due to the water led to the decreasing of the rising and the development of the sprouts of the seeds of two kinds of radishes.

2. Under indirect effect due to the water the low doses of $\gamma$-irradiation lowered, and $\alpha$-irradiation enhanced the negative effect of a 40 -fold attenuated geomagnetic field on the growth characteristics of the highest plants 
seeds, i.e. ionized radiation was the dominant factor in the combined effect with the weakening geomagnetic field.

3. The oxidation-reduction potential of water increased as magnetic induction attenuated pointing to a natural decline in hypomagnetic chamber.

4. The effect of enhancing doses of $\alpha$-irradiation revealed a non-linear polymodal character of water ORP value alterations, which kept even 20 minutes later after the time of exposition.

5. The effect of enhancing doses of $\gamma$-irradiation registered less range of water ORP value changing in comparison with the effect of $\alpha$-particles, which reflected on the growth characteristics of seeds of the highest plants.

6 . The combined action of hypomagnetic field and $\alpha$ - and $\gamma$-irradiation in low doses changed water oxidation-reduction properties depending on the type, absorbed dose of radiation source, and reflected on the physiological characteristics of seeds of the highest plants.

7. In our opinion, namely water is the main component in the effects of weakening of magnetic field and ionized radiation on bio-objects, which acts due to the alterations of the properties and structural content of water.

\section{References}

[1] Koronovsky, N.V. (1996) Magnetic Field of Geological Past of Earth. System of Life Activity Support, 5, 56-63.

[2] Moisa, S.S., Zotin, A.A. and Tsetlin, V.V. (2015) The Embryonic Development of Great Ramshorn Planorbarius corneus under the Hypomagnetic Field. American Journal of Life Sciences, 3, 25-31.

[3] Vorontsov, I.V., Zhiljaev, E.G., Karpov, V.N. and Ushakov, I.B. (2002) Low Radiation Effects and Human Health. Voronezhskii State University, Moscow.

[4] Grigoriev, Yu.G., Popov, V.I., Shafirkin, A.V., et al. (1986) Somatic Effects of Chronic Gamma-Irradiation. Atomizdat, Moscow.

[5] Kuzin, A.M. (1995) The Ideas of Radiation Hormezis in Atom Century. Nauka, Moscow.

[6] Pelevina, I.I., Aleschenko, A.V., Gotlib, V.Ya., et al. (2005) Reaction of Blood Lymphocytes of Individual with Somatic Diseases on the Radiation Effect in Low Doses. Journal of Radiation Biology, 45, 412-415.

[7] Luckey, T.D. (1980) Hormesis with Ionizing Radiation. CRC Press, Boca Raton.

[8] Ushakov, I.B., Tsetlin, V.V. and Moisa, S.S. (2012) Reaction of Water and Living Systems under the Chronic Effect of Ionized Radiation in Low Doses. Journal of Biomedical Science and Engineering, 5, 771-778. http://dx.doi.org/10.4236/jbise.2012.512A097

[9] Tsetlin, V.V., Zenin, S.V., Golovkina, T.V., et al. (2003) Role of Water Medium in the Mechanism of the Effect of Super-Low Doses of Ionized radiation. Journal of Bio-Medical Technologies and Radio-Electronic, 12, 20-25.

[10] Tsetlin, V.V. (2010) Studies into Water Reactions to Variations of Cosmophysical and Geophysical Factors of the Environment. Journal of Avia-Cosmic and Ecological Medicine, 6, 26-31.

[11] Tsetlin, V.V. and Fineshtein, G.S. (2012) About the Effect of Cosmophysic, Geophysic and Radiation Factors on the Electro-Physic and Biological Water Properties. Journal of Metaphysics, 2, 81-99.

[12] Bahir, I.M. (1999) The Determination of the Terms “Water" and "Solution” Conforming to the Technology of Electrochemical Activation. Electrochemical Activation in Medicine, Agriculture and Industry, 14, 41-47.

[13] Rassadkin, Yu P. (2008) Water Usual and Unusual. Moscow.

[14] Rusov, V.D. and Zeletsova, T.N. (2002) Introduction in Non-Linear Theory of Low Doses of Ionized Radiation. Odessa.

[15] Chukova, Yu.P. (2002) Low-Level Influence Effects. Ales, Moscow.

[16] Zatsepina, G.N. (1974) Water Properties and Structure. MGU, Moscow.

[17] Knorre, D.G., Krylova, D.F. and Muzykantov, V.S. (1990) Physical Chemistry. Vysshaya Shkola, Moscow. 


\section{Submit or recommend next manuscript to SCIRP and we will provide best service for you:}

Accepting pre-submission inquiries through Email, Facebook, Linkedin, Twitter, etc A wide selection of journals (inclusive of 9 subjects, more than 200 journals)

Providing a 24-hour high-quality service

User-friendly online submission system

Fair and swift peer-review system

Efficient typesetting and proofreading procedure

Display of the result of downloads and visits, as well as the number of cited articles

Maximum dissemination of your research work

Submit your manuscript at: http://papersubmission.scirp.org/ 